\title{
Radioactive Iodine-Refractory Differentiated Thyroid Cancer and Redifferentiation Therapy
}

\author{
Jierui Liu ${ }^{1,2,3}$, Yanqing Liu ${ }^{1,2}$, Yansong Lin ${ }^{1,2,3}$, Jun Liang ${ }^{4}$ \\ ${ }^{1}$ Department of Nuclear Medicine, Peking Union Medical College Hospital; ${ }^{2}$ Beijing Key Laboratory of Molecular Targeted \\ Diagnosis and Therapy in Nuclear Medicine, Beijing; ${ }^{3}$ Department of Oncology, The Affiliated Hospital of Qingdao University, \\ Qingdao; ${ }^{4}$ Department of Oncology, Peking University International Hospital, Beijing, China
}

The retained functionality of the sodium iodide symporter (NIS) expressed in differentiated thyroid cancer (DTC) cells allows the further utilization of post-surgical radioactive iodine (RAI) therapy, which is an effective treatment for reducing the risk of recurrence, and even the mortality, of DTC. Whereas, the dedifferentiation of DTC could influence the expression of functional NIS, thereby reducing the efficacy of RAI therapy in advanced DTC. Genetic alternations (such as BRAF and the rearranged during transfection $[\mathrm{RET}]$ /papillary thyroid cancer [PTC] rearrangement) have been widely reported to be prominently responsible for the onset, progression, and dedifferentiation of PTC, mainly through activating the mitogen-activated protein kinase (MAPK) and phosphoinositide 3-kinase (PI3K) signaling cascades. These genetic alternations have been suggested to associate with the reduced expression of iodide-handling genes in thyroid cancer, especially the NIS gene, disabling iodine uptake and causing resistance to RAI therapy. Recently, novel and promising approaches aiming at various targets have been attempted to restore the expression of these iodine-metabolizing genes and enhance iodine uptake through in vitro studies and studies of RAI-refractory (RAIR)-DTC patients. In this review, we discuss the regulation of NIS, known mechanisms of dedifferentiation including the MAPK and PI3K pathways, and the current status of redifferentiation therapy for RAIR-DTC patients.

Keywords: Thyroid neoplasms; Sodium-iodide symporter; Isotopes

\section{INTRODUCTION}

Thyroid cancer has emerged as a striking health issue over recent decades because of its gradually increasing incidence worldwide. The global incidence of thyroid cancer is 6.7 per 100,000, and the number of newly diagnosed likely cases in China has exceeded 190,000 (194,232 cases) [1]. Papillary thyroid cancer

Received: 12 August 2019, Revised: 29 August 2019, Accepted: 4 September 2019

Corresponding authors: Yansong Lin

Department of Nuclear Medicine, Peking Union Medical College Hospital,

No. 1 Shuaifuyuan, Wangfujing St, Dongcheng, Beijing 100730, China

Tel: +86-10-69155610, Fax: +86-10-69155610, E-mail: linys@pumch.cn

Jun Liang

Department of Oncology, Peking University International Hospital, No.1 Life Park

St., Zhongguancun Life Science Park, Haidian, Beijing 102206, China

Tel: +86-10-69006624, Fax: +86-10-69006082, E-mail: liangjun1959@aliyun.com
(PTC), follicular thyroid cancer, and Hürthle cell cancer are derived from follicular cells; thus, they are collectively characterized as differentiated thyroid cancer (DTC), and account for more than $90 \%$ of all thyroid malignancies [2,3]. Although most DTC cases have a quite favorable prognosis after standard therapeutic approaches, including surgery, selective radioactive iodine (RAI) therapy, and thyroid stimulating hormone (TSH) suppres-

\section{Copyright $(\subset 2019$ Korean Endocrine Society}

This is an Open Access article distributed under the terms of the Creative Commons Attribution Non-Commercial License (http://creativecommons.org/ licenses/by-nc/4.0/) which permits unrestricted non-commercial use, distribution, and reproduction in any medium, provided the original work is properly cited. 
sive therapy, the risk of local recurrence and distant metastasis may be up to $20 \%$ and $10 \%$, respectively. Among these patients, two-thirds show initial or gradual loss of the ability of iodine uptake due to the dysfunction, and even loss, of sodium iodide symporter (NIS) expression in the basal membrane, indicating a status of dedifferentiation known as RAI-refractory DTC (RAIRDTC), which is of major clinical concern because its 10-year survival rate is less than $10 \%$ [4].

Genetic alterations are the fundamental drivers for the tumorigenesis and pathogenesis of thyroid cancer, aberrantly activating the mitogen-activated protein kinase (MAPK) and phosphoinositide 3-kinase (PI3K) pathways [5,6]. These alternations are also known to be associated with the silencing of various thyroid iodine-metabolizing genes, especially solute carrier family 5 member 5 (SLC5A5), which encodes NIS, thus resulting in the failure of RAI therapy [7-9]. Multiple agents have been investigated in attempts to restore NIS expression and to enhance RAI uptake in RAIR-DTC patients. This review aimed to summarize the regulation of NIS, the possible mechanism of NIS downregulation, and the potential of redifferentiation therapy for RAIR-DTC.

\section{PHYSIOLOGICAL REGULATION OF NIS EXPRESSION}

Iodide, as an essential component, is utilized by follicular cells to synthesize thyroid hormone in the normal thyroid. As it is expressed on the basal membrane, NIS provides the physiological basis for the active transport of iodide into the follicular cells of the thyroid [10]. DTC cells can retain similar functions to those of follicular cells, such as iodine uptake and iodination [11,12], which allows RAI therapy to be the mainstay for the treatment of intermediate and high-risk DTC after surgery [13]. To destroy residual or potential subclinical lesions, RAI therapy could improve disease-specific survival and progression-free survival [14]. Hence, the function or expression of NIS in DTC cells is crucial for the efficacy of RAI therapy in such patients.

Functional NIS expression can be regulated at both the transcriptional and post-translational levels. As the predominant regulator of NIS expression, TSH is primarily involved at the translational level. After the binding of TSH with the TSH receptor, adenylyl cyclase is stimulated through the Gs-protein, which in turn increases the expression of cyclic adenosine monophosphate (cAMP). cAMP then induces NIS transcription by activating several signaling pathways that could stimulate the NIS upstream enhancer (NUE). It is now known that the human
NUE consists of paired box gene-8 (PAX8; thyroid-specific transcription factor) binding site and cAMP-response element like site, both of which are important for the integrated activity of the NUE [15]. As illustrated in Fig. 1, cAMP can stimulate the NUE through both protein kinase A (PKA)-independent and -dependent pathways. Through redox effector factor-1 (Ref-1), the PKA-independent pathway, PAX8 is subsequently stimulated to bind to the NUE, leading to the activation of NUE [15-18], and this pathway plays a key role in the differentiation of the thyroid [19]. Through the PKA-dependent pathway, the activated PKA could phosphorylate the cAMP-responsive element modulator, enhancing NUE activity [20,21].

TSH-independent mechanisms also regulate NIS expression, which mainly include three pathways influencing the binding of

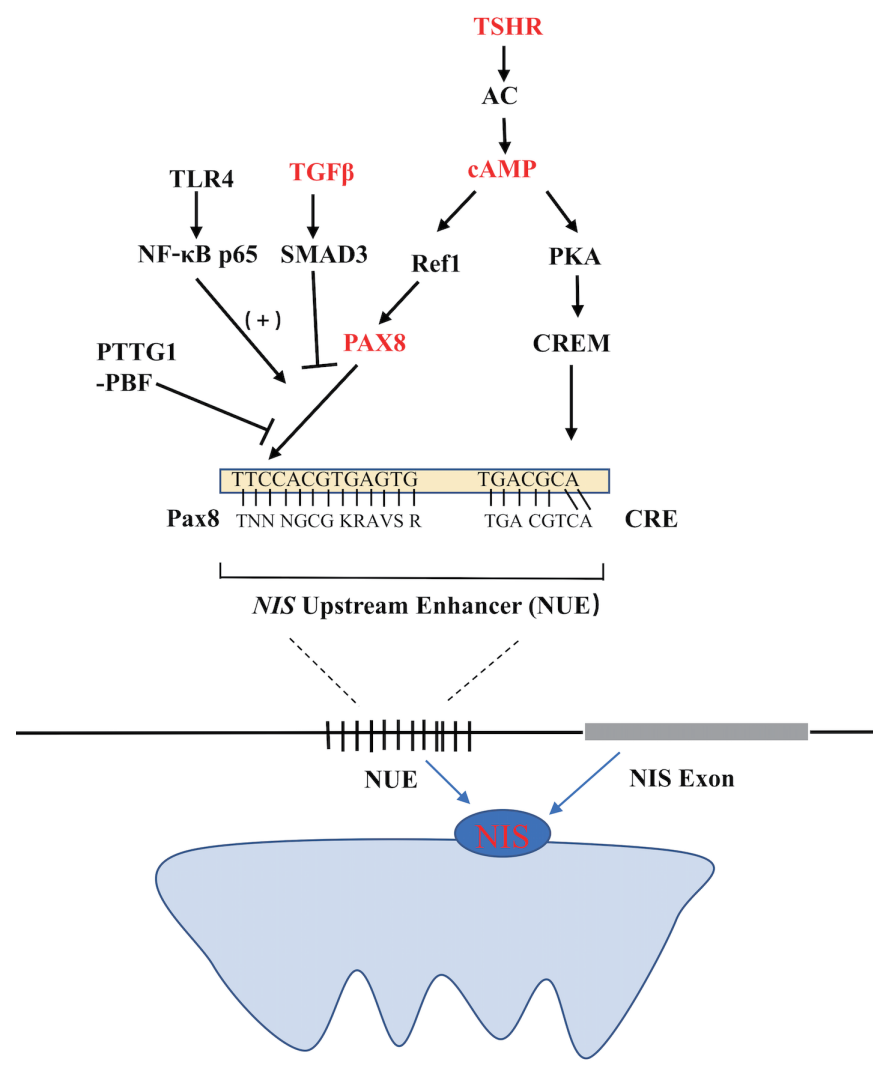

Fig. 1. Regulation of the sodium iodide symporter (NIS) upstream enhancer (NUE) at the transcriptional level in thyroid cells. TSHR, thyroid stimulating hormone receptor; AC, adenylyl cyclase; cAMP, cyclic adenosine monophosphate; PKA, protein kinase A; CRE, cAMP-response element; CREM, CRE-modulator; Ref1, apurinic apyrimidinic endonuclease redox effector factor-1; Pax8, paired box gene-8; TGF $\beta$, transforming growth factor $\beta$; TLR4, Toll-like receptor 4 ; NF- $\kappa \mathrm{B}, \mathrm{p} 65$, a member of the class II nuclear factor $\kappa$-light-chain-enhancer of activated B cells, p65; PTTG1, pituitary tumor-transforming gene-1; PBF, PTTG1-binding factor. 
PAX8 to NUE. First, in the transforming growth factor $\beta$ (TGF $\beta$ )-SMAD signaling pathway, TGF $\beta$ activates the downstream Smad3 and subsequently inhibits the binding of PAX8 to NUE, significantly decreasing NIS mRNA expression in thyroid cells $[9,16]$. Second, in the Toll-like receptor (TLR)-nuclear factor $\kappa$-light-chain-enhancer of activated B cells (NF- $\kappa \mathrm{B})$ signaling pathway, the TLR activates the downstream NF- $\kappa B$, which further interacts with PAX8, activating NIS transcription via the NUE $[22,23]$. Third, in the pituitary tumor-transforming gene-1 product (PTTG1)-binding factor (PBF) complex, the PTTG1 and the PBF could interfere with the binding of PAX8 to the NUE, thus suppressing the expression of NIS (Fig. 1) [24-26]. Saez et al. [26] reported that increased PTTG1 expression could reduce the efficacy of RAI therapy in thyroid cancer.

Concerning post-translational regulation, abundant NIS expression may mis-localize in the intracellular compartment rather than the cell membrane [27]. This abnormal membrane targeting of NIS could disable iodide transport and result in the reduced uptake and accumulation of RAI in thyroid cancer cells, inducing the probable failure of RAI therapy in a subset of DTC.

\section{KNOWN PATHWAYS DOWNREGULATING THE EXPRESSION OF NIS}

\section{MAPK/ERK pathway}

The MAPK pathway has been well recognized and established in the regulation of cell proliferation, dedifferentiation, and survival [28-30], particularly for PTC. Among the signal molecules of this pathway, BRAF mutations and the rearranged during transfection (RET)/PTC rearrangement are frequently detected in PTC, which often exhibit as exclusive mutations in such patients $[5,31,32]$. The $B R A F^{\mathrm{V} 600 \mathrm{E}}$ mutation, as a prominently prevalent oncogene, is critical for the initiation and/or progression of PTC through aberrantly activating the MAPK signaling pathway, which can downregulate the expression of thyroid iodide-handling genes, especially NIS, and thus induce the dedifferentiation of PTC [28,33-36]. Increasing evidence has demonstrated a strong association between the $B R A F^{\mathrm{V} 600 \mathrm{E}}$ mutation and the loss of RAI-avidity in PTC [37-40], which could provide a reasonable explanation for the failure of RAI therapy in $B R A F^{\mathrm{V} 600 \mathrm{E}}$-mutant PTC.

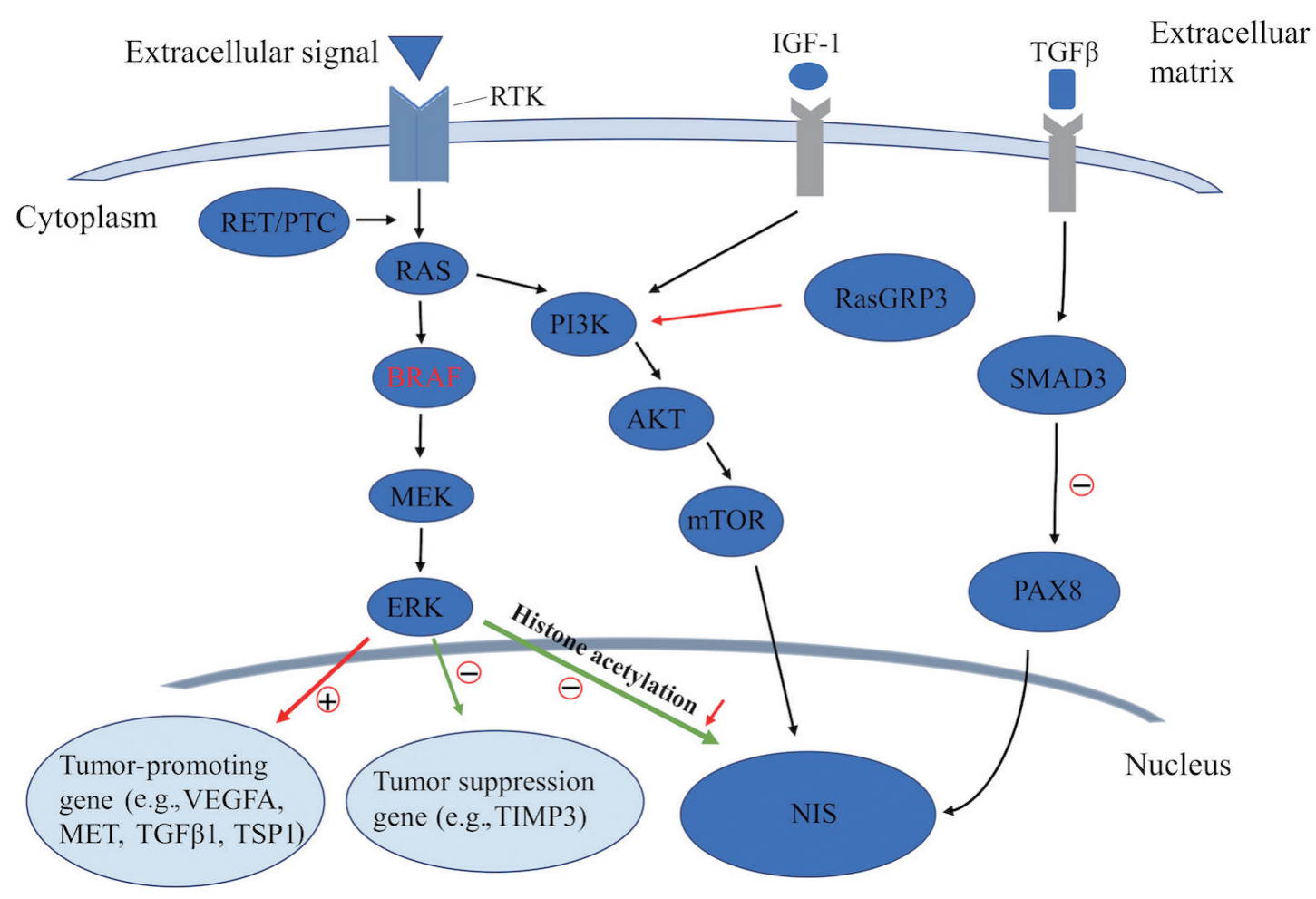

Fig. 2. Known pathways involved in the regulation of sodium iodide symporter (NIS) in thyroid cancer. RTK, receptor tyrosine kinase; IGF1 , insulin-like growth factor-1; TGF $\beta$, transforming growth factor $\beta$; PTC, papillary thyroid cancer; PI3K, phosphoinositide 3-kinase; RasGRP3, Ras guanyl releasing protein 3; PAX8, paired box gene-8; MEK, mitogen-activated extracellular signal-regulated kinase; ERK, extracellular regulated protein kinase; mTOR, mechanistic target of rapamycin; VEGFA, vascular endothelial growth factor A; MET, mesenchymal to epithelial transition factor; TSP1, thrombospondin 1; TIMP3, tissue inhibitor of metalloproteinases 3. 
As an important epigenetic event, histone acetylation plays a fundamental role in the regulation of gene transcription [41,42]. Through aberrant activation of the MAPK pathway, histone acetylation at the promoter of the gene encoding NIS could be downregulated, which is considered as one of the key molecular events involving the aberrant silencing of thyroid iodide-handling genes [5,43-46]. Meanwhile, the $B R A F^{\mathrm{V} 600 \mathrm{E}}$ mutation can also upregulate the expression of tumor-promoting genes (e.g., vascular endothelial growth factor A [VEGFA], mesenchymal to epithelial transition factor [MET], TGF $\beta 1$, and thrombospondin 1 [TSP1]) [9,34,47-49] and downregulate the expression of tumor suppressor genes (e.g., tissue inhibitor of metalloproteinases 3 [TIMP3], solute carrier family 5 member 8 [SLC5A8], and death-associated protein kinase $1[D A P K 1])$ [50], which are important constituents of the tumor microenvironment. It is noteworthy that the autocrine TGF $\beta$ loop could play a role in aberrant NIS expression. Riesco-Eizaguirre et al. [9] and Costamagna et al. [16] reported that the $B R A F^{\mathrm{V} 600 \mathrm{E}}$ mutation could induce the secretion of TGF $\beta$, which subsequently stimulated SMAD3 and impaired PAX8, causing a decrease of NIS expression. As this process was independent of the MAPK pathway, their result undoubtedly indicates that TGF $\beta$ could be considered as a candidate therapeutic target for the restoration of NIS expression in patients with advanced DTC (Fig. 2).

It was recently recognized that telomerase reverse transcriptase (TERT) promoter (TERTp) mutations are particularly prevalent in aggressive thyroid cancers, especially $B R A F^{\mathrm{V} 600 \mathrm{E}}-\mathrm{mu}-$ tant PTC, but are virtually undetectable in benign thyroid neoplasms [51,52]. TERTp mutations were demonstrated to be associated with aggressive tumor behavior and poor prognosis in thyroid cancer [53-55], and were also observed to be correlated with the reduction of RAI uptake in distant metastatic lesions of PTC [56], which suggested that TERTp mutations may play a role in the dedifferentiation of thyroid cancer. It was also reported that the duet alternation of $B R A F^{\mathrm{V} 600 \mathrm{E}}$ and TERTp mutations have a robust synergistic effect on the progression and poor clinical outcomes of PTC. Recently, Liu et al. [57] revealed that the $B R A F^{\mathrm{V} 600 \mathrm{E}} / \mathrm{MAPK}$ pathway could phosphorylate and activate fructooligosaccharide, which in turn acted as a transcription factor to activate the GA binding protein transcription factor subunit beta $(G A B P B)$ promoter, increasing $G A B P B$ expression and leading to the formation of the GA binding protein transcription factor subunit alpha (GABPA)-GABPB complex, thus activating the mutant TERT promoter and upregulating TERT expression. The discovery of TERTp mutations and the genetic duet of coexisting mutations could afford promising molecular targets for the salvage therapy of RAIR-PTC.

Concerning the RET/PTC rearrangement, evidence about its impact on the dedifferentiation of DTC remains limited. Trapasso et al. [58] and Wang et al. [59] reported that alternation of $\mathrm{RET} / \mathrm{PTC}$ in the thyroid cell line could decrease the expression of thyroid differentiation markers, such as TSH receptor, TPO, NIS, and thyroglobulin. Furthermore, exogenous RET/PTC could significantly suppress the expression of PAX8 and the activity of PKA, leading to reduced NIS expression $[8,58]$.

\section{PI3K/AKT pathway}

In addition to the MAPK pathway, the PI3K/AKT pathway also plays a fundamental role in controlling both cell proliferation and differentiation in DTC. In recent years, several genetic alterations activating the PI3K pathway have been identified in thyroid cancer, which was also shown to downregulate iodide-handling genes in thyroid cells $[5,60]$. Song et al. [61] recently reported that, also by this pathway, mutation of Ras guanyl releasing protein 3 (RasGRP3) decreased the expression of NIS and the TSH receptor in metastases of RAIR-DTC. Furthermore, the PI3K pathway could be activated by multiple growth factors such as insulin/insulin-like growth factor-1 (IGF-1) and epidermal growth factor [62]. Garcia and Santisteban [63] reported that IGF-1 could inhibit TSH-dependent NIS expression and reduce the iodide uptake in fisher rat thyroid cell line-5 (FRTL-5) rat thyroid cells through activating the PI3K/AKT pathway (Fig. 2). It has been shown that inhibition of the PI3K pathway by LY294002 could significantly increase the expression of NIS mRNA in rat thyroid cells and PTC cells, which, from another aspect, indicated the important role of the PI3K pathway in regulating NIS-mediated iodide accumulation in thyroid cancer [64]. The serine-threonine protein kinase mechanistic target of rapamycin (mTOR), which is located downstream of the PI3K/ AKT pathway, has been identified as a regulator of cellular metabolism and proliferation. Souza et al. [65] reported that mTOR inhibition not only regulated cell survival, but also increased RAI uptake in both in vitro and in vivo studies.

\section{REDIFFERENTIATION THERAPY FOR RAIR-DTC}

During the past decade, although great progress has been achieved in the treatment of RAIR-DTC with the application of multi-kinase inhibitors (MKIs), therapeutic options for these patients are still limited. As RAI resistance is predominantly due to the dedifferentiation of DTC, redifferentiation therapy 
followed by RAI therapy undoubtedly is a promising alternative option for RAIR-DTC patients. Several agents, including retinoic acid (RA) [66], peroxisome proliferator-activated receptor gamma (PPAR $\gamma$ ) agonists [67], and histone deacetylase (HDAC) inhibitors [68], have been tried to modulate the NIS gene at the transcription level, but displayed limited clinical value in redifferentiation therapy for patients with RAIR-DTC. Recent studies using drugs that selectively inhibit the MAPK and PI3K pathways showed promising results for restoring the expression of the gene encoding NIS and improving the response to RAI therapy in RAIR-DTC, such as mitogen-activated extracellular signal-regulated kinase (MEK)/RAF inhibitors $[69,70]$, $\mathrm{PI} 3 \mathrm{~K} / \mathrm{mTOR}$ inhibitors [64] and receptor tyrosine kinase (RTK) inhibitors [71], which were used to restore NIS expression by suppressing the signaling pathways in such patients.

\section{Agents modulating NIS at the gene transcriptional level}

RA is a biologically active metabolite of vitamin A that play key roles in cell differentiation and proliferation. RA has been used for redifferentiation treatment of thyroid cancer, exerting its effects via retinoid receptors, $R A$ receptors ( $R A R$ ), or retinoid $X$ receptors (RXR). Studies have shown that NIS mRNA expression was upregulated by RA stimulation in human follicular thyroid carcinoma cell lines [72]. Several early small cohort studies showed that $40 \%$ to $50 \%$ of patients with RAIR-DTC experienced renewed RAI uptake after RA treatment [73-75]. However, such promising results could not be repeated by subsequent studies, which disappointingly indicated that only $6 \%$ to $20 \%$ of patients showed increased RAI uptake after RA administration $[66,76,77]$. A recent open-label, non-randomized phase II trial reported even more disappointing results, with only one patient (1/16) showing increased RAI uptake after RA administration [66].

The PPAR $\gamma$ is a nuclear receptor which can regulate the proliferation and redifferentiation of the cell through forming a heterodimer with RXR [78,79]. Pieces of evidence have shown that the rosiglitazone, a PPAR $\gamma$ agonist agent for the redifferentiation treatment of thyroid cancer, increased NIS mRNA expression and RAI uptake in thyroid cells $[80,81]$. However, the results of a phase II clinical trial were disappointing, as $25 \%$ (5/20) of the patients displayed positive RAI uptake after rosiglitazone treatment, but no clinical response in long-term follow-up [67].

HDAC is an enzyme that deacetylates histones, which could silence the expression of NIS in thyroid cancer [5]. HDAC inhibitors were found to increase the expression of NIS mRNA at the epigenetic level in an in vitro study [82]. Preclinical studies have shown renewed RAI uptake after treatment with various HDAC inhibitors, such as suberoylanilide hydroxamic acid (SAHA), depsipeptide (romidepsin), and valproic acid. Nevertheless, their independent significance in clinical trials was limited. Kelly et al. [83] reported that only one (1/3) advanced thyroid cancer patient showed increased RAI uptake after SAHA administration. In a phase I clinical trial of romidepsin, no positive RAI uptake was detected by RAI scintigraphy among 11 enrolled RAIR-DTC patients [84]. Furthermore, the results of a phase II clinical trial using romidepsin showed that only two patients experienced increased RAI uptake and no major response was observed in 20 patients with RAIR-DTC [68]. Nilubol et al. [85] performed a phase II clinical trial to evaluate the effect of valproic acid, without renewed RAI uptake.

\section{MAPK inhibitors}

The independent administration of retinoids, romidepsin, and rosiglitazone was shown to have limited clinical application in redifferentiation therapy for RAIR-DTC. With the development of molecular and cellular biology, multiple novel targets have been revealed, which could afford new options for the redifferentiation therapy of RAIR-DTC. Evidence has shown that agents selectively inhibiting the MAPK pathway, such as BRAF or MEK inhibitors, could induce thyroid gene expression and restore RAI uptake in thyroid cancer cells $[43,86]$. The results of a clinical trial performed by Ho et al. [69] revealed that, after treatment with selumetinib, a selective MEK inhibitor, ${ }^{124} \mathrm{I}$ uptake was increased in RAIR-PTC patients, which indicated the restoration of NIS expression in these patients. Meanwhile, it was interesting that the efficacy of selumetinib was better in patients with an NRAS mutation than those with the $B R A F^{\mathrm{v} 600 \mathrm{E}}$ mutation [69]. Furthermore, the prior phase II clinical trial of selumetinib seemed to suggest that $B R A F^{\mathrm{V} 600 \mathrm{E}}$ mutant patients exhibited longer median progression-free survival than patients with $B R A F$ wild-type tumors [87]. This suggested a potential relationship between the therapeutic efficacy of selumetinib and genetic alterations. However, it was disappointing that the subsequent phase III trial of selumetinib failed to reconfirm its effect in the restoration of RAI uptake, which suggested that further studies are needed to explore the efficacy of MEK inhibitors in the redifferentiation of RAIR-DTC.

Another phase II clinical trial showed that dabrafenib, a BRAF inhibitor, increased RAI uptake in $60 \%(6 / 10)$ of $B R A F$ mutant RAIR-DTC patients, with two of the six achieving partial response after subsequent RAI therapy [88], which surpassed selumetinib in the treatment of BRAF-mutated RAIR- 
DTC. Vemurafenib, another BRAF inhibitor, could also restore RAI uptake in a subset of $B R A F$-mutant RAIR-DTC patients, which was likely due to the upregulation of thyroid-specific gene expression via inhibition of the MAPK pathway [70]. Earlier studies showed that HDAC inhibitors (such as SAHA) alone could induce the expression of NIS and faint RAI uptake in thyroid cancer cells $[89,90]$; gratifyingly, a recent study reported that combined treatment with an HDAC inhibitor and a MAPK inhibitor (dabrafenib and selumetinib) showed a robust redifferentiation effect in $B R A F^{\mathrm{V} 600 \mathrm{E}}$-mutated thyroid cancer cells [91]. This result suggests that the combined administration of HDAC and/or MAPK inhibitors might be a promising choice to improve the efficacy of redifferentiation therapy in RAIRDTC patients. Furthermore, Nagarajah et al. [92] reported that extracellular regulated protein kinase (ERK) inhibitors could significantly increase the accumulation of ${ }^{124} \mathrm{I}$ in $B R A F^{\mathrm{V} 600 \mathrm{E}}$-mutant PTC cells, indicating that ERK inhibitors may be a potential candidate for redifferentiation therapy in $B R A F^{\mathrm{V} 600 \mathrm{E}}$-mutant PTC.

\section{PI3K inhibitors}

As mentioned above, the aberrant activation of the PI3K/AKT/ mTOR pathway could downregulate NIS expression [5], indicating that this pathway may be a potential therapeutic target for the redifferentiation therapy of RAIR-DTC. LY294002, a PI3K inhibitor, significantly upregulated the expression of NIS mRNA and improved iodide uptake through induction of PAX8 in DTC cell lines [64,93]. Moreover, the inhibition of AKT also exhibited an increase of iodide uptake by mediating the expression of NIS in thyroid cells [93]. Plantinga et al. [94] reported that an mTOR inhibitor could induce iodine uptake through increasing thyroid transcription factor 1 (TTF1) expression in an in vitro study. Nevertheless, findings have not been reported from several in vivo studies that were initiated to evaluate the changes of iodine uptake by suppressing the PI3K pathway in RAIR-DTC patients. Hence, further studies might be necessary to elucidate the impact of PI3K/AKT/mTOR pathway inhibitors on the redifferentiation of RAIR-DTC.

\section{RTK inhibitors}

RTKs, such as vascular endothelial growth factor receptor (VEGFR), RET, platelet-derived growth factor receptors (PDGFRs), and human epidermal growth factor receptor (HER), are also crucial molecules inducing the dedifferentiation of DTC. By aberrantly causing the MAPK pathway to rebound, RTKs, such as HER3, could also lead to resistance to MKIs in RAIR-
DTC patients [95]. Agents targeting RTKs have been recently investigated for the redifferentiation and salvage therapy of MKI-resistant RAIR-DTC. Cheng et al. [71] reported that combination therapy of BRAF/MEK inhibitors (dabrafenib/selumetinib) with an HER inhibitor (lapatinib) could upregulate NIS expression and suppress the MAPK pathway without a rebound phenomenon, and the corresponding phase I trial is now underway. The above-mentioned results may afford us a new prospective for the redifferentiation therapy of RAIR-DTC, as well as alternative salvage therapy for MKI-resistant advanced thyroid cancer.

\section{CONCLUSIONS}

The expression of NIS allows RAI therapy to be a highly effective management strategy for DTC, especially in metastatic DTC patients. Genetic alternations could reduce the expression of NIS and lead to the dedifferentiation of DTC, mainly through activation of the MAPK and PI3K pathways in thyroid cancer, causing a RAIR state that represents a life-threatening clinical situation. Efforts have been made to restore NIS expression and enhance RAI avidity in RAIR-DTC. Agents for dedifferentiation therapy at the transcriptional level have yielded limited clinical impact in clinical trials. Kinase inhibitors targeting the MAPK or PI3K pathways have shown promising effects in redifferentiation therapy and shed light on future combination therapy between either kinase inhibitors with different targets or kinase inhibitors and RAI in the management of RAIR-DTC.

\section{CONFLICTS OF INTEREST}

No potential conflict of interest relevant to this article was reported.

\section{ACKNOWLEDGMENTS}

This review was supported by the National Natural Sciences Foundation of China (81771875 and 81571714), the Medicine and Technology Innovation Project of the Chinese Academy of Medical Science (grant number 2016-12M-2-006), and the Thyroid Study Group Project of the Asia Oceania Research Initiative Network (AORIN).

\section{ORCID}

Jierui Liu https://orcid.org/0000-0003-0528-5407 
Yansong Lin https://orcid.org/0000-0001-6095-4728

Jun Liang https://orcid.org/0000-0001-7624-4413

\section{REFERENCES}

1. Ferlay J, Colombet M, Soerjomataram I, Mathers C, Parkin DM, Pineros M, et al. Estimating the global cancer incidence and mortality in 2018: GLOBOCAN sources and methods. Int J Cancer 2019;144:1941-53.

2. Mao Y, Xing M. Recent incidences and differential trends of thyroid cancer in the USA. Endocr Relat Cancer 2016;23:31322.

3. Lim H, Devesa SS, Sosa JA, Check D, Kitahara CM. Trends in thyroid cancer incidence and mortality in the United States, 1974-2013. JAMA 2017;317:1338-48.

4. Durante C, Haddy N, Baudin E, Leboulleux S, Hartl D, Travagli JP, et al. Long-term outcome of 444 patients with distant metastases from papillary and follicular thyroid carcinoma: benefits and limits of radioiodine therapy. J Clin Endocrinol Metab 2006;91:2892-9.

5. Xing M. Molecular pathogenesis and mechanisms of thyroid cancer. Nat Rev Cancer 2013;13:184-99.

6. Zaballos MA, Santisteban P. Key signaling pathways in thyroid cancer. J Endocrinol 2017;235:R43-61.

7. Xing M. BRAF mutation in thyroid cancer. Endocr Relat Cancer 2005;12:245-62.

8. Venkateswaran A, Marsee DK, Green SH, Jhiang SM. Forskolin, 8-Br-3',5'-cyclic adenosine 5'-monophosphate, and catalytic protein kinase A expression in the nucleus increase radioiodide uptake and sodium/iodide symporter protein levels in RET/PTC1-expressing cells. J Clin Endocrinol Metab 2004;89:6168-72.

9. Riesco-Eizaguirre G, Rodriguez I, De la Vieja A, Costamagna E, Carrasco N, Nistal M, et al. The BRAFV600E oncogene induces transforming growth factor beta secretion leading to sodium iodide symporter repression and increased malignancy in thyroid cancer. Cancer Res 2009;69:8317-25.

10. Riesco-Eizaguirre G, Santisteban P. A perspective view of sodium iodide symporter research and its clinical implications. Eur J Endocrinol 2006;155:495-512.

11. Castro MR, Bergert ER, Goellner JR, Hay ID, Morris JC. Immunohistochemical analysis of sodium iodide symporter expression in metastatic differentiated thyroid cancer: correlation with radioiodine uptake. J Clin Endocrinol Metab 2001;86:5627-32.

12. Wapnir IL, van de Rijn M, Nowels K, Amenta PS, Walton K,
Montgomery K, et al. Immunohistochemical profile of the sodium/iodide symporter in thyroid, breast, and other carcinomas using high density tissue microarrays and conventional sections. J Clin Endocrinol Metab 2003;88:1880-8.

13. American Thyroid Association (ATA) Guidelines Taskforce on Thyroid Nodules and Differentiated Thyroid Cancer, Cooper DS, Doherty GM, Haugen BR, Kloos RT, Lee SL, et al. Revised American Thyroid Association management guidelines for patients with thyroid nodules and differentiated thyroid cancer. Thyroid 2009;19:1167-214.

14. Tuttle RM, Ahuja S, Avram AM, Bernet VJ, Bourguet P, Daniels GH, et al. Controversies, consensus, and collaboration in the use of (131)I therapy in differentiated thyroid cancer: a joint statement from the American Thyroid Association, the European Association of Nuclear Medicine, the Society of Nuclear Medicine and Molecular Imaging, and the European Thyroid Association. Thyroid 2019;29:461-70.

15. Taki K, Kogai T, Kanamoto Y, Hershman JM, Brent GA. A thyroid-specific far-upstream enhancer in the human sodium/iodide symporter gene requires Pax- 8 binding and cyclic adenosine 3',5'-monophosphate response element-like sequence binding proteins for full activity and is differentially regulated in normal and thyroid cancer cells. Mol Endocrinol 2002;16:2266-82.

16. Costamagna E, Garcia B, Santisteban P. The functional interaction between the paired domain transcription factor Pax8 and Smad3 is involved in transforming growth factorbeta repression of the sodium/iodide symporter gene. J Biol Chem 2004;279:3439-46.

17. Kambe F, Nomura Y, Okamoto T, Seo H. Redox regulation of thyroid-transcription factors, Pax-8 and TTF-1, is involved in their increased DNA-binding activities by thyrotropin in rat thyroid FRTL-5 cells. Mol Endocrinol 1996;10:801-12.

18. Tell G, Pellizzari L, Cimarosti D, Pucillo C, Damante G. Ref-1 controls pax-8 DNA-binding activity. Biochem Biophys Res Commun 1998;252:178-83.

19. Mansouri A, Chowdhury K, Gruss P. Follicular cells of the thyroid gland require Pax8 gene function. Nat Genet 1998; 19:87-90.

20. Chun JT, Di Dato V, D'Andrea B, Zannini M, Di Lauro R. The CRE-like element inside the 5'-upstream region of the rat sodium/iodide symporter gene interacts with diverse classes of b-Zip molecules that regulate transcriptional activities through strong synergy with Pax-8. Mol Endocrinol 2004;18:2817-29.

21. Fenton MS, Marion KM, Hershman JM. Identification of cy- 
clic adenosine 3',5'-monophosphate response element modulator as an activator of the human sodium/iodide symporter upstream enhancer. Endocrinology 2008;149:2592-606.

22. Nicola JP, Velez ML, Lucero AM, Fozzatti L, Pellizas CG, Masini-Repiso AM. Functional toll-like receptor 4 conferring lipopolysaccharide responsiveness is expressed in thyroid cells. Endocrinology 2009;150:500-8.

23. Nicola JP, Nazar M, Mascanfroni ID, Pellizas CG, MasiniRepiso AM. NF-kappaB p65 subunit mediates lipopolysaccharide-induced $\mathrm{Na}(+) / \mathrm{I}(-)$ symporter gene expression by involving functional interaction with the paired domain transcription factor Pax8. Mol Endocrinol 2010;24:1846-62.

24. Boelaert K, Smith VE, Stratford AL, Kogai T, Tannahill LA, Watkinson JC, et al. PTTG and PBF repress the human sodium iodide symporter. Oncogene 2007;26:4344-56.

25. Stratford AL, Boelaert K, Tannahill LA, Kim DS, Warfield A, Eggo MC, et al. Pituitary tumor transforming gene binding factor: a novel transforming gene in thyroid tumorigenesis. J Clin Endocrinol Metab 2005;90:4341-9.

26. Saez C, Martinez-Brocca MA, Castilla C, Soto A, Navarro E, Tortolero M, et al. Prognostic significance of human pituitary tumor-transforming gene immunohistochemical expression in differentiated thyroid cancer. J Clin Endocrinol Metab 2006;91:1404-9.

27. Kogai T, Brent GA. The sodium iodide symporter (NIS): regulation and approaches to targeting for cancer therapeutics. Pharmacol Ther 2012;135:355-70.

28. Xing M. BRAF mutation in papillary thyroid cancer: pathogenic role, molecular bases, and clinical implications. Endocr Rev 2007;28:742-62.

29. Cohen Y, Xing M, Mambo E, Guo Z, Wu G, Trink B, et al. BRAF mutation in papillary thyroid carcinoma. J Natl Cancer Inst 2003;95:625-7.

30. Liu D, Liu Z, Condouris S, Xing M. BRAF V600E maintains proliferation, transformation, and tumorigenicity of BRAF-mutant papillary thyroid cancer cells. J Clin Endocrinol Metab 2007;92:2264-71.

31. Kimura ET, Nikiforova MN, Zhu Z, Knauf JA, Nikiforov YE, Fagin JA. High prevalence of BRAF mutations in thyroid cancer: genetic evidence for constitutive activation of the RET/PTC-RAS-BRAF signaling pathway in papillary thyroid carcinoma. Cancer Res 2003;63:1454-7.

32. Soares P, Trovisco V, Rocha AS, Lima J, Castro P, Preto A, et al. BRAF mutations and RET/PTC rearrangements are alternative events in the etiopathogenesis of PTC. Oncogene 2003;22:4578-80.
33. Kim TH, Park YJ, Lim JA, Ahn HY, Lee EK, Lee YJ, et al. The association of the BRAF(V600E) mutation with prognostic factors and poor clinical outcome in papillary thyroid cancer: a meta-analysis. Cancer 2012;118:1764-73.

34. Giordano TJ, Kuick R, Thomas DG, Misek DE, Vinco M, Sanders D, et al. Molecular classification of papillary thyroid carcinoma: distinct BRAF, RAS, and RET/PTC mutation-specific gene expression profiles discovered by DNA microarray analysis. Oncogene 2005;24:6646-56.

35. Riesco-Eizaguirre G, Gutierrez-Martinez P, Garcia-Cabezas MA, Nistal M, Santisteban P. The oncogene BRAF V600E is associated with a high risk of recurrence and less differentiated papillary thyroid carcinoma due to the impairment of $\mathrm{Na}+/ \mathrm{I}-$ targeting to the membrane. Endocr Relat Cancer 2006;13:257-69.

36. Mian C, Barollo S, Pennelli G, Pavan N, Rugge M, Pelizzo $\mathrm{MR}$, et al. Molecular characteristics in papillary thyroid cancers (PTCs) with no 131I uptake. Clin Endocrinol (Oxf) 2008;68:108-16.

37. Xing M, Westra WH, Tufano RP, Cohen Y, Rosenbaum E, Rhoden KJ, et al. BRAF mutation predicts a poorer clinical prognosis for papillary thyroid cancer. J Clin Endocrinol Metab 2005;90:6373-9.

38. Ricarte-Filho JC, Ryder M, Chitale DA, Rivera M, Heguy A, Ladanyi $\mathrm{M}$, et al. Mutational profile of advanced primary and metastatic radioactive iodine-refractory thyroid cancers reveals distinct pathogenetic roles for BRAF, PIK3CA, and AKT1. Cancer Res 2009;69:4885-93.

39. Barollo S, Pennelli G, Vianello F, Watutantrige Fernando S, Negro I, Merante Boschin I, et al. BRAF in primary and recurrent papillary thyroid cancers: the relationship with (131) I and 2-[(18)F]fluoro-2-deoxy-D-glucose uptake ability. Eur J Endocrinol 2010;163:659-63.

40. Yang K, Wang H, Liang Z, Liang J, Li F, Lin Y. BRAFV600E mutation associated with non-radioiodine-avid status in distant metastatic papillary thyroid carcinoma. Clin Nucl Med 2014;39:675-9.

41. Barneda-Zahonero B, Parra M. Histone deacetylases and cancer. Mol Oncol 2012;6:579-89.

42. Horikoshi M. Histone acetylation: from code to web and router via intrinsically disordered regions. Curr Pharm Des 2013;19:5019-42.

43. Hou P, Bojdani E, Xing M. Induction of thyroid gene expression and radioiodine uptake in thyroid cancer cells by targeting major signaling pathways. J Clin Endocrinol Metab 2010;95:820-8. 
44. Durante C, Puxeddu E, Ferretti E, Morisi R, Moretti S, Bruno $\mathrm{R}$, et al. BRAF mutations in papillary thyroid carcinomas inhibit genes involved in iodine metabolism. J Clin Endocrinol Metab 2007;92:2840-3.

45. Di Cristofaro J, Silvy M, Lanteaume A, Marcy M, Carayon P, De Micco C. Expression of tpo mRNA in thyroid tumors: quantitative PCR analysis and correlation with alterations of ret, Braf, ras and pax8 genes. Endocr Relat Cancer 2006; 13:485-95.

46. Zhang Z, Liu D, Murugan AK, Liu Z, Xing M. Histone deacetylation of NIS promoter underlies BRAF V600E-promoted NIS silencing in thyroid cancer. Endocr Relat Cancer 2014;21:161-73.

47. Jo YS, Li S, Song JH, Kwon KH, Lee JC, Rha SY, et al. Influence of the BRAF V600E mutation on expression of vascular endothelial growth factor in papillary thyroid cancer. $\mathrm{J}$ Clin Endocrinol Metab 2006;91:3667-70.

48. Nucera C, Porrello A, Antonello ZA, Mekel M, Nehs MA, Giordano TJ, et al. B-Raf(V600E) and thrombospondin-1 promote thyroid cancer progression. Proc Natl Acad Sci U S A 2010;107:10649-54.

49. Knauf JA, Sartor MA, Medvedovic M, Lundsmith E, Ryder M, Salzano M, et al. Progression of BRAF-induced thyroid cancer is associated with epithelial-mesenchymal transition requiring concomitant MAP kinase and TGF $\beta$ signaling. Oncogene 2011;30:3153-62.

50. Hu S, Liu D, Tufano RP, Carson KA, Rosenbaum E, Cohen Y, et al. Association of aberrant methylation of tumor suppressor genes with tumor aggressiveness and BRAF mutation in papillary thyroid cancer. Int J Cancer 2006;119:2322-9.

51. Liu R, Xing M. TERT promoter mutations in thyroid cancer. Endocr Relat Cancer 2016;23:R143-55.

52. Alzahrani AS, Alsaadi R, Murugan AK, Sadiq BB. TERT promoter mutations in thyroid cancer. Horm Cancer 2016;7: 165-77.

53. Sohn SY, Park WY, Shin HT, Bae JS, Ki CS, Oh YL, et al. Highly concordant key genetic alterations in primary tumors and matched distant metastases in differentiated thyroid cancer. Thyroid 2016;26:672-82.

54. Bu R, Siraj AK, Divya SP, Kong Y, Parvathareddy SK, AlRasheed $\mathrm{M}$, et al. Telomerase reverse transcriptase mutations are independent predictor of disease-free survival in Middle Eastern papillary thyroid cancer. Int J Cancer 2018; 142:2028-39.

55. Liu R, Bishop J, Zhu G, Zhang T, Ladenson PW, Xing M. Mortality risk stratification by combining BRAF V600E and
TERT promoter mutations in papillary thyroid cancer: genetic duet of BRAF and TERT promoter mutations in thyroid cancer mortality. JAMA Oncol 2017;3:202-8.

56. Yang X, Li J, Li X, Liang Z, Gao W, Liang J, et al. TERT promoter mutation predicts radioiodine-refractory character in distant metastatic differentiated thyroid cancer. J Nucl Med 2017;58:258-65.

57. Liu R, Zhang T, Zhu G, Xing M. Regulation of mutant TERT by BRAF V600E/MAP kinase pathway through FOS/GABP in human cancer. Nat Commun 2018;9:579.

58. Trapasso F, Iuliano R, Chiefari E, Arturi F, Stella A, Filetti S, et al. Iodide symporter gene expression in normal and transformed rat thyroid cells. Eur J Endocrinol 1999;140:447-51.

59. Wang J, Knauf JA, Basu S, Puxeddu E, Kuroda H, Santoro $\mathrm{M}$, et al. Conditional expression of RET/PTC induces a weak oncogenic drive in thyroid PCCL3 cells and inhibits thyrotropin action at multiple levels. Mol Endocrinol 2003; 17:1425-36.

60. Xing M. Genetic alterations in the phosphatidylinositol-3 kinase/Akt pathway in thyroid cancer. Thyroid 2010;20:697706.

61. Song J, Qiu W, Deng X, Qiu Z, Fan Y, Yang Z. A somatic mutation of RasGRP3 decreases $\mathrm{Na}(+) / \mathrm{I}(-)$ symporter expression in metastases of radioactive iodine-refractory thyroid cancer by stimulating the Akt signaling pathway. Am J Cancer Res 2018;8:1847-55.

62. Kimura T, Van Keymeulen A, Golstein J, Fusco A, Dumont JE, Roger PP. Regulation of thyroid cell proliferation by TSH and other factors: a critical evaluation of in vitro models. Endocr Rev 2001;22:631-56.

63. Garcia B, Santisteban P. PI3K is involved in the IGF-I inhibition of TSH-induced sodium/iodide symporter gene expression. Mol Endocrinol 2002;16:342-52.

64. Kogai T, Sajid-Crockett S, Newmarch LS, Liu YY, Brent GA. Phosphoinositide-3-kinase inhibition induces sodium/ iodide symporter expression in rat thyroid cells and human papillary thyroid cancer cells. J Endocrinol 2008;199:24352.

65. de Souza EC, Padron AS, Braga WM, de Andrade BM, Vaisman M, Nasciutti LE, et al. MTOR downregulates iodide uptake in thyrocytes. J Endocrinol 2010;206:113-20.

66. Short SC, Suovuori A, Cook G, Vivian G, Harmer C. A phase II study using retinoids as redifferentiation agents to increase iodine uptake in metastatic thyroid cancer. Clin Oncol (R Coll Radiol) 2004;16:569-74.

67. Kebebew E, Lindsay S, Clark OH, Woeber KA, Hawkins R, 
Greenspan FS. Results of rosiglitazone therapy in patients with thyroglobulin-positive and radioiodine-negative advanced differentiated thyroid cancer. Thyroid 2009;19:953-6.

68. Sherman EJ, Su YB, Lyall A, Schoder H, Fury MG, Ghossein RA, et al. Evaluation of romidepsin for clinical activity and radioactive iodine reuptake in radioactive iodine-refractory thyroid carcinoma. Thyroid 2013;23:593-9.

69. Ho AL, Grewal RK, Leboeuf R, Sherman EJ, Pfister DG, Deandreis D, et al. Selumetinib-enhanced radioiodine uptake in advanced thyroid cancer. N Engl J Med 2013;368: 623-32.

70. Dunn LA, Sherman EJ, Baxi SS, Tchekmedyian V, Grewal RK, Larson SM, et al. Vemurafenib redifferentiation of BRAF mutant, RAI-refractory thyroid cancers. J Clin Endocrinol Metab 2019;104:1417-28.

71. Cheng L, Jin Y, Liu M, Ruan M, Chen L. HER inhibitor promotes BRAF/MEK inhibitor-induced redifferentiation in papillary thyroid cancer harboring BRAFV600E. Oncotarget 2017;8:19843-54.

72. Schmutzler C, Schmitt TL, Glaser F, Loos U, Kohrle J. The promoter of the human sodium/iodide-symporter gene responds to retinoic acid. Mol Cell Endocrinol 2002;189:14555.

73. Grunwald F, Menzel C, Bender H, Palmedo H, Otte R, Fimmers $\mathrm{R}$, et al. Redifferentiation therapy-induced radioiodine uptake in thyroid cancer. J Nucl Med 1998;39:1903-6.

74. Simon D, Koehrle J, Reiners C, Boerner AR, Schmutzler C, Mainz K, et al. Redifferentiation therapy with retinoids: therapeutic option for advanced follicular and papillary thyroid carcinoma. World J Surg 1998;22:569-74.

75. Simon D, Korber C, Krausch M, Segering J, Groth P, Gorges $\mathrm{R}$, et al. Clinical impact of retinoids in redifferentiation therapy of advanced thyroid cancer: final results of a pilot study. Eur J Nucl Med Mol Imaging 2002;29:775-82.

76. Kim WG, Kim EY, Kim TY, Ryu JS, Hong SJ, Kim WB, et al. Redifferentiation therapy with 13 -cis retinoic acids in radioiodine-resistant thyroid cancer. Endocr J 2009;56:10512.

77. Courbon F, Zerdoud S, Bastie D, Archambaud F, Hoff M, Eche N, et al. Defective efficacy of retinoic acid treatment in patients with metastatic thyroid carcinoma. Thyroid 2006;16: 1025-31.

78. Corton JC, Anderson SP, Stauber A. Central role of peroxisome proliferator-activated receptors in the actions of peroxisome proliferators. Annu Rev Pharmacol Toxicol 2000; 40:491-518.
79. Roberts-Thomson SJ. Peroxisome proliferator-activated receptors in tumorigenesis: targets of tumour promotion and treatment. Immunol Cell Biol 2000;78:436-41.

80. Frohlich E, Machicao F, Wahl R. Action of thiazolidinediones on differentiation, proliferation and apoptosis of normal and transformed thyrocytes in culture. Endocr Relat Cancer 2005;12:291-303.

81. Park JW, Zarnegar R, Kanauchi H, Wong MG, Hyun WC, Ginzinger DG, et al. Troglitazone, the peroxisome proliferator-activated receptor-gamma agonist, induces antiproliferation and redifferentiation in human thyroid cancer cell lines. Thyroid 2005;15:222-31.

82. Kitazono M, Robey R, Zhan Z, Sarlis NJ, Skarulis MC, Aikou T, et al. Low concentrations of the histone deacetylase inhibitor, depsipeptide (FR901228), increase expression of the $\mathrm{Na}(+) / \mathrm{I}(-)$ symporter and iodine accumulation in poorly differentiated thyroid carcinoma cells. J Clin Endocrinol Metab 2001;86:3430-5.

83. Kelly WK, O’Connor OA, Krug LM, Chiao JH, Heaney M, Curley T, et al. Phase I study of an oral histone deacetylase inhibitor, suberoylanilide hydroxamic acid, in patients with advanced cancer. J Clin Oncol 2005;23:3923-31.

84. Amiri-Kordestani L, Luchenko V, Peer CJ, Ghafourian K, Reynolds J, Draper D, et al. Phase I trial of a new schedule of romidepsin in patients with advanced cancers. Clin Cancer Res 2013;19:4499-507.

85. Nilubol N, Merkel R, Yang L, Patel D, Reynolds JC, Sadowski SM, et al. A phase II trial of valproic acid in patients with advanced, radioiodine-resistant thyroid cancers of follicular cell origin. Clin Endocrinol (Oxf) 2017;86:128-33.

86. Liu D, Hu S, Hou P, Jiang D, Condouris S, Xing M. Suppression of BRAF/MEK/MAP kinase pathway restores expression of iodide-metabolizing genes in thyroid cells expressing the V600E BRAF mutant. Clin Cancer Res 2007; 13:1341-9.

87. Hayes DN, Lucas AS, Tanvetyanon T, Krzyzanowska MK, Chung CH, Murphy BA, et al. Phase II efficacy and pharmacogenomic study of Selumetinib (AZD6244; ARRY-142886) in iodine-131 refractory papillary thyroid carcinoma with or without follicular elements. Clin Cancer Res 2012;18:205665.

88. Rothenberg SM, McFadden DG, Palmer EL, Daniels GH, Wirth LJ. Redifferentiation of iodine-refractory BRAF V600E-mutant metastatic papillary thyroid cancer with dabrafenib. Clin Cancer Res 2015;21:1028-35.

89. Furuya F, Shimura H, Suzuki H, Taki K, Ohta K, Haraguchi 
$\mathrm{K}$, et al. Histone deacetylase inhibitors restore radioiodide uptake and retention in poorly differentiated and anaplastic thyroid cancer cells by expression of the sodium/iodide symporter thyroperoxidase and thyroglobulin. Endocrinology 2004;145:2865-75.

90. Pugliese M, Fortunati N, Germano A, Asioli S, Marano F, Palestini N, et al. Histone deacetylase inhibition affects sodium iodide symporter expression and induces 131I cytotoxicity in anaplastic thyroid cancer cells. Thyroid 2013;23: 838-46.

91. Fu H, Cheng L, Jin Y, Cheng L, Liu M, Chen L. MAPK inhibitors enhance HDAC inhibitor-induced redifferentiation in papillary thyroid cancer cells harboring BRAF (V600E): an in vitro study. Mol Ther Oncolytics 2019;12:235-45.

92. Nagarajah J, Le M, Knauf JA, Ferrandino G, MonteroConde C, Pillarsetty N, et al. Sustained ERK inhibition maximizes responses of BrafV600E thyroid cancers to radioiodine. J Clin Invest 2016;126:4119-24.

93. Liu YY, Zhang X, Ringel MD, Jhiang SM. Modulation of sodium iodide symporter expression and function by LY294002, Akti-1/2 and rapamycin in thyroid cells. Endocr Relat Cancer 2012;19:291-304.

94. Plantinga TS, Heinhuis B, Gerrits D, Netea MG, Joosten LA, Hermus AR, et al. mTOR inhibition promotes TTF1dependent redifferentiation and restores iodine uptake in thyroid carcinoma cell lines. J Clin Endocrinol Metab 2014; 99:E1368-75.

95. Montero-Conde C, Ruiz-Llorente S, Dominguez JM, Knauf JA, Viale A, Sherman EJ, et al. Relief of feedback inhibition of HER3 transcription by RAF and MEK inhibitors attenuates their antitumor effects in BRAF-mutant thyroid carcinomas. Cancer Discov 2013;3:520-33. 